\title{
Effects of reproductive status and high ambient temperatures on the body temperature of a free-ranging basoendotherm
}

\author{
Danielle L. Levesque · Kerileigh D. Lobban • \\ Barry G. Lovegrove
}

Received: 24 March 2014 / Revised: 30 June 2014 / Accepted: 13 August 2014

(C) Springer-Verlag Berlin Heidelberg 2014

\begin{abstract}
Tenrecs (Order Afrosoricida) exhibit some of the lowest body temperatures $\left(T_{\mathrm{b}}\right)$ of any eutherian mammal. They also have a high level of variability in both active and resting $T_{\mathrm{b}} \mathrm{s}$ and, at least in cool temperatures in captivity, frequently employ both short- and long-term torpor. The use of heterothermy by captive animals is, however, generally reduced during gestation and lactation. We present data long-term $T_{\mathrm{b}}$ recordings collected from free-ranging S. setosus over the course of two reproductive seasons. In general, reproductive females had slightly higher $\left(\sim 32^{\circ} \mathrm{C}\right)$ and less variable $T_{\mathrm{b}}$, whereas non-reproductive females and males showed both a higher propensity for torpor as well as lower $\left(\sim 30.5^{\circ} \mathrm{C}\right)$ and more variable rest-phase $T_{\mathrm{b}} \mathrm{s}$. Torpor expression defined using traditional means (using a threshold or cut-off $T_{\mathrm{b}}$ ) was much lower than predicted based on the high degree of heterothermy in captive tenrecs. However, torpor defined in this manner is likely to be underestimated in habitats where ambient temperature is close to $T_{\mathrm{b}}$. Our results caution against inferring metabolic states from $T_{\mathrm{b}}$ alone and lend support to the recent call to define torpor in free-ranging animals based on mechanistic
\end{abstract}

Communicated by G. Heldmaier.

Electronic supplementary material The online version of this article (doi:10.1007/s00360-014-0858-4) contains supplementary material, which is available to authorized users.

D. L. Levesque $(\triangle)$

Institute of Biodiversity and Environmental Conservation, Universiti Malaysia Sarawak, 94300 Kota Samarahan,

Sarawak, Malaysia

e-mail: 1ldanielle@ibec.unimas.my

D. L. Levesque · K. D. Lobban · B. G. Lovegrove School of Life Sciences, University of KwaZulu-Natal, P/Bag X01, Scottsville 3209, South Africa and not descriptive variables. In addition, lower variability in $T_{\mathrm{b}}$ observed during gestation and lactation confirms that homeothermy is essential for reproduction in this species and probably for basoendothermic mammals in general. The relatively low costs of maintaining homeothermy in a sub-tropical environment might help shed light on how homeothermy could have evolved incrementally from an ancestral heterothermic condition.

Keywords Evolution of endothermy · Parental care · Thermoregulation · Reproduction · Heterothermy · Torpor · Madagascar · Tropics · Setifer setosus $\cdot$ Tenrec
Abbreviations
$\mathrm{T}_{\mathrm{a}} \quad$ Ambient temperature measured via a black body apparatus
$T_{\mathrm{b}} \quad$ Core body temperature
$T_{\text {soil }} \quad$ Ambient temperature measured at a depth of $250 \mathrm{~mm}$ below the surface
$T_{\text {tree }} \quad$ Ambient temperature measured in a tree cavity
$\Delta T \quad$ Temperature differential $\left(T_{\mathrm{b}}-T_{\mathrm{a}}\right)$
$T_{\text {bmax }}$ Average of the 5 highest $T_{\mathrm{b}}$ data for each day
$T_{\text {bmin }} \quad$ Average of the 5 lowest $T_{\mathrm{b}}$ data for each day
$\Delta T_{\mathrm{b}} \quad$ Difference between the daily maximum and mini- mum $T_{\mathrm{b}}$

\section{Introduction}

The reconstructed mammalian ancestor is postulated to have been small bodied, nocturnal and insectivorous (Crompton et al. 1978; Luo 2007; Gerkema et al. 2013; O'Leary et al. 2013), and is hypothesised to have possessed the capacity for prolonged periods of metabolic downregulation, torpor (Lovegrove 2012a). The plesiomorphy of 